\title{
siRNA as a sponge
}

New nanostructure delivers potent dose of short interfering RNA

When researchers discuss the potential of RNA interference to knock down genes of target cells in vivo, they trip up on a common question: how can enough of the necessary short interfering RNAs (siRNAs) be made to reach the intended cells? A suite of nanoparticles and other strategies have already been tried as delivery vehicles, but none works perfectly. They are often destroyed by the body, and packing enough of the RNA molecules into them is difficult. Researchers led by Paula Hammond at the Massachusetts Institute of Technology now demonstrate another strategy, called a microsponge. Rather than encapsulate RNA molecules inside a delivery device, they fashion a delivery vehicle made from RNA molecules themselves.

To make the sponge, Hammond and colleagues took inspiration from 'DNA origami' and other techniques to create nanostructures from nucleotides. They found a way to make exceptionally long RNA strands comprising an estimated hundreds of thousands to millions of nucleotides. These strands were also designed to be cleaved by cells' RNA-processing machinery into siRNAs. Within about 20 hours in solution, the RNA strands fold themselves into spherical, spongelike structures, all about 2 micrometers across. Such self-assembled crystalline superstructures have not previously been described for any other RNA polymer, says Hammond.

The sponges were readily taken up by cultured cancer cells, where they could knock down the expression of a reporter gene by about $42 \%$ (as measured by luminescence). The sponges could also knock down reporter expression when injected into tumors grown in mice.
Sponges offer several potential advantages for RNA delivery, says Hammond. Other delivery systems contain multiple components and are often plagued by high manufacturing costs as well as risks of toxicity and immunogenicity. The crystalline form of the sponge protects the RNA from degradation before it enters cells. Whereas other nanoparticles usually contain less than 5\% RNA, the sponges are made entirely of RNA, explains Hammond. "You need about 1,000 typical nanoparticles to contain the same amount of siRNA as one siRNA microsponge." Hammond is currently working to modify sponges to facilitate delivery through the bloodstream and to specific types of tissue.

\section{Monya Baker}

\section{RESEARCH PAPERS}

Lee, J.B. et al. Self-assembled RNA interference microsponges for efficient siRNA delivery. Nat. Mater. advance online publication (26 February 2012). 